\title{
CORONAL JETS, MAGNETIC TOPOLOGIES, AND THE PRODUCTION OF INTERPLANETARY ELECTRON STREAMS
}

\author{
C. $\mathrm{LI}^{1,2}$, S. A. Matthews ${ }^{1}$, L. van Driel-Gesztelyi ${ }^{1,3,4}$, J. Sun $^{1}$, And C. J. Owen ${ }^{1}$ \\ ${ }^{1}$ Mullard Space Science Laboratory, University College London, Dorking, Surrey RH5 6NT, UK; cl2@mssl.ucl.ac.uk \\ ${ }^{2}$ Department of Astronomy, Nanjing University, Nanjing 210093, China \\ ${ }^{3}$ Observatoire de Paris, LESIA, UMR 8109 (CNRS), 92195 Meudon Cedex, France \\ ${ }^{4}$ Konkoly Observatory of Hungarian Academy of Sciences, Budapest, Hungary \\ Received 2011 March 7; accepted 2011 April 15; published 2011 June 14
}

\begin{abstract}
We investigate the acceleration source of the impulsive solar energetic particle (SEP) events on 2007 January 24. Combining the in situ electron measurements and remote-sensing solar observations, as well as the calculated magnetic fields obtained from a potential-field source-surface model, we demonstrate that the jets associated with the hard X-ray flares and type-III radio bursts, rather than the slow and partial coronal mass ejections, are closely related to the production of interplanetary electron streams. The jets, originated from the well-connected active region (AR 10939) whose magnetic polarity structure favors the eruption, are observed to be forming in a coronal site, extending to a few solar radii, and having a good temporal correlation with the electron solar release. The open-field lines near the jet site are rooted in a negative polarity, along which energetic particles escape from the flaring AR to the near-Earth space, consistent with the in situ electron pitch angle distribution. The analysis enables us to propose a coronal magnetic topology relating the impulsive SEP events to their solar source.
\end{abstract}

Key words: Sun: flares - Sun: magnetic topology - Sun: particle emission

Online-only material: color figures

\section{INTRODUCTION}

A class of solar energetic particle (SEP) events, which are associated with "impulsive" $\mathrm{X}$-ray flares and type-III radio bursts, show distinct enhancement of $e / p$ ratio and in some cases high- $Z$ elemental abundance (e.g., ${ }^{3} \mathrm{He}$ and $\mathrm{Fe}$ ). Such energetic electrons and heavy ions are believed to be originated from flaring active regions (ARs), separating from large coronal mass ejection (CME) and shock-related "gradual" events (see reviews by Reames 1999; Kallenrode 2003; Mason 2007). Apart from the irreducible requirement of a western hemisphere AR where magnetic and plasma processes preferentially energize and release particles (Mason et al. 2009), we still do not clearly identify the solar source of the impulsive SEP events.

Recently, Wang et al. (2006) and Nitta et al. (2008) proposed that jets seen in extreme-ultraviolet (EUV) and sometimes in white-light (WL) images are closely related to the ${ }^{3} \mathrm{He}$-rich SEP events. The jets may be explained as expanding loops reconnecting with large-scale unipolar magnetic fields (Shibata et al. 1994), which are open to interplanetary space for energetic particles to be observed at 1 AU. However, Kahler et al. (2001) and Pick et al. (2006) found some unusual cases of ${ }^{3} \mathrm{He}$-rich SEP events associated with fast and narrow CMEs, making the source identification more complex. A possibility is suggested that particles are accelerated close to the jets or plasmoids, which move upward from magnetic reconnection sites and might appear as narrow CMEs in coronagraphs (Pick et al. 2006). These recent progresses in identifying the solar source still leave unsolved questions. Do the jets or CMEs in association with the impulsive SEP events play a critical role in particle acceleration (Mason et al. 2009)? How does the magnetic topology in the source region relate solar activities to the in situ SEP dynamics (Li et al. 2010)? To answer the questions, a careful comparison of in situ particle measurements and remote- sensing solar observations as well as the modeling of coronal magnetic fields should be carried out.

In this study, we present a cross-disciplinary investigation of the impulsive SEP events observed on 2007 January 24, by using energetic electron data to constrain the in situ SEP dynamics (for instance, timing and distribution), which are much more accurate than with low-energy ion data, and the multi-wavelength imaging data to detect the details of the solar activities. We also apply the velocity map, magnetogram, and the potential-field extrapolation to interpret the magnetic morphology in the source region. Our purpose is to clarify the links between jet eruption, coronal magnetic topology, and the production of interplanetary electron streams.

\section{IN SITU MEASUREMENTS}

Two successive electron events were observed on 2007 January 24 by the WIND three-dimensional Plasma and Energetic Particles instrument (3DP; Lin et al. 1995) and the Electron, Proton, and Alpha Monitor (EPAM; Gold et al. 1998) on board the Advanced Composition Explorer (ACE), both of which are orbiting the Sun-Earth L1 Lagrange point. The WIND/3DP provides electron measurements with the electrostatic analyzers from $\sim 0.5$ to $28 \mathrm{keV}$ and with the solid-state telescopes from 27 to $\sim 300 \mathrm{keV}$, which suffered a data gap during the events. Compensating for the data gap, the ACE/EPAM measures electrons with the Composition Aperture (CA), whose look direction is oriented $60^{\circ}$ from the spacecraft spin axis and so is known as the CA60 telescope, in the energy range of $38-315 \mathrm{keV}$.

Figure 1 shows the electron intensity profiles observed from $\sim 1$ to $\sim 200 \mathrm{keV}$ by the WIND/3DP and ACE/EPAM instruments, respectively, shown in panels (a) and (b), together

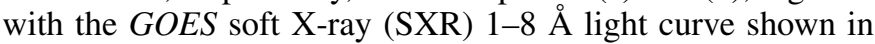
panel (c). Two successive flux enhancements, marked as event 1 and event 2, were recorded. Both events show clear velocity dispersion (injection and peak times are later for lower energies), 

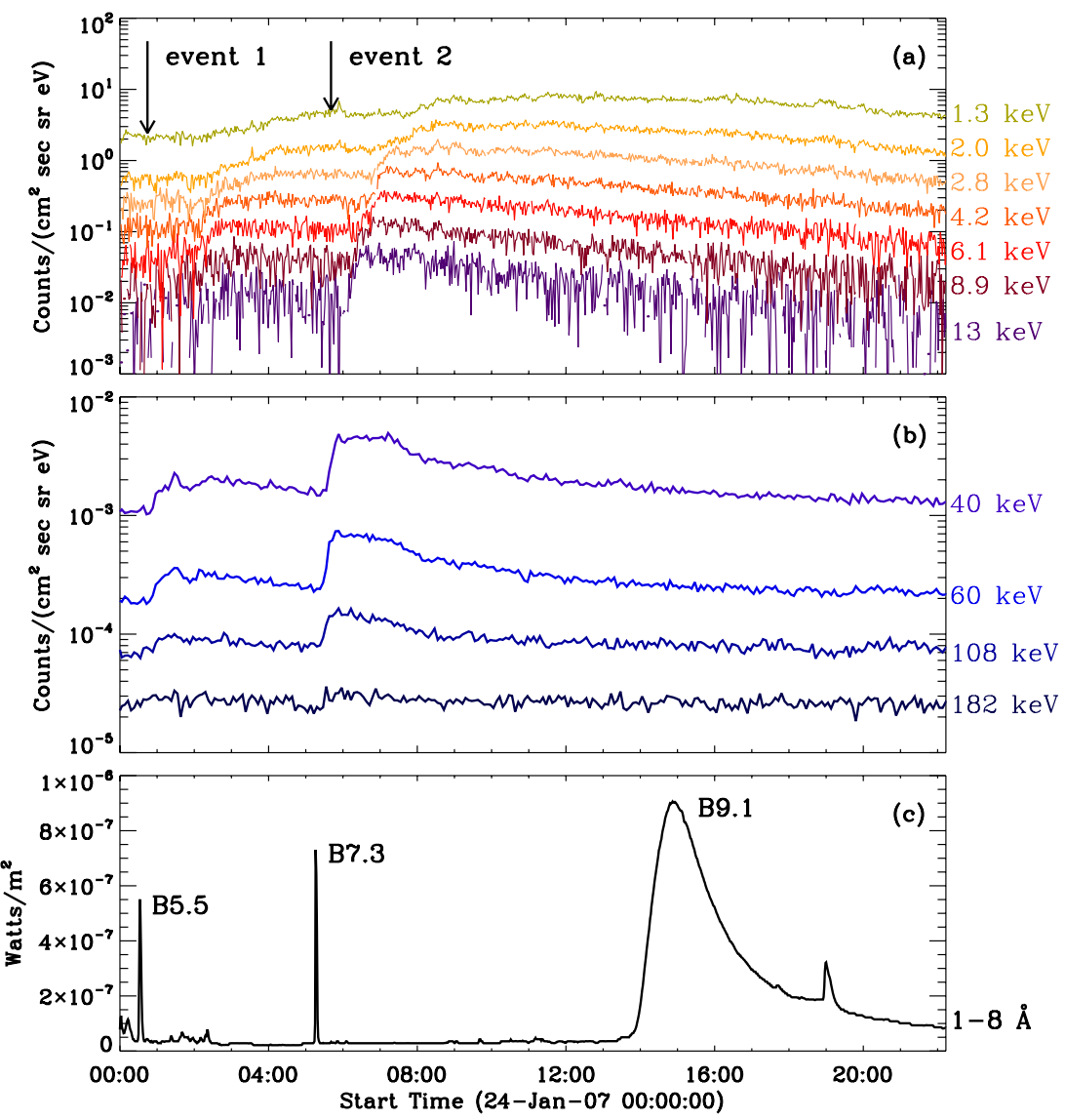

Figure 1. Impulsive electron events observed on 2007 January 24. (a) Intensity profiles of in situ energetic electrons from $\sim 1$ to $13 \mathrm{keV}$ detected by the WIND/3DP, and (b) from 38 to $315 \mathrm{keV}$ by the ACE/EPAM, compared with (c) the GOES SXR 1-8 A light curve.

(A color version of this figure is available in the online journal.)

which is the typical identification of the solar origin. They correspond to the impulsive B5.5 and B7.3 flares, respectively, which erupted from AR 10939 on the west hemisphere. When the flares occurred, the position of this AR was roughly at S06W60, near the footpoints of interplanetary magnetic field (IMF) lines connecting the Sun to the near-Earth space, facilitating energetic particles escape to the in situ spacecraft. The larger B9.1 flare, even though accompanied by a relatively speaking fast (projected speed of $785 \mathrm{~km} \mathrm{~s}^{-1}$ ) and wide (angular width of $147^{\circ}$ ) CME, was produced by an eastern AR located at S04E110, too far from the well-connected region, therefore could not be related to an SEP event.

Unfortunately, there were severe data gaps of solar imaging observations in the time window of event 1 . Therefore, we mainly focus on the event 2 in the following study. To determine the solar release time, assuming electrons travel along the IMF lines at a speed of $v(\epsilon)$ in energy channel $\epsilon$ with no scattering (Krucker et al. 1999), we apply a linear fit to $t_{\text {sol }}(\epsilon)=t_{\mathrm{AU}}(\epsilon)-L / v(\epsilon)$, where $L$ is the IMF path length from the acceleration site on the Sun to the in situ spacecraft, $t_{\mathrm{AU}}(\epsilon)$ and $t_{\mathrm{sol}}(\epsilon)$ are the rise time of electron flux at $1 \mathrm{AU}$ and the release time at the Sun in energy channel $\epsilon$, respectively. Then the extrapolated electron solar release time $t_{\text {sol }}(\epsilon \rightarrow \infty)$ is 05:19 UT \pm 5 minutes, and the inferred IMF path length $L$ is $1.3 \pm 0.2 \mathrm{AU}$. To further confirm this evaluation, adapting the solar wind speed of $\sim 380 \mathrm{~km} \mathrm{~s}^{-1}$ during this event, the IMF path length is calculated by solution of the IMF equation deduced from the solar wind model (Parker 1958) to be $\sim 1.19$ AU.
Taking $v(\epsilon)$ to be $\sim 0.5 c$ in the ACE/EPAM energy channel $\epsilon$ of $60 \mathrm{keV}$, this channel leads to a lower bound on the electron solar release time (Haggerty \& Roelof 2003). This is reasonable since electrons actually undergo interplanetary scattering, then the electron solar release time is derived to be $\sim 05: 20$ UT. The results are consistent with the previous evaluation.

To understand the anisotropy of the in situ energetic electrons requires an examination of particle transport direction relative to the magnetic field. The Low Energy Foil Spectrometer oriented at $60^{\circ}$ to the spin axis (LEFS60) of the $A C E / E P A M$ provides measurements of electron fluxes in eight angular sectors, whose spatial orientations projected onto the unit sphere are shown in the left panel of Figure 2. The magnetic field vector at the peak time of electron fluxes around 05:55 UT is obtained from the $A C E /$ magnetometer (MAG) in a geocentric solar ecliptic (GSE) coordinate. Then the electron pitch-angle distribution (PAD) is derived and shown in the right panel of Figure 2. The letters correspond to the eight angular sectors in the $42-65 \mathrm{keV}$ channel. The distribution is normalized and plotted against the pitch-cosine. Except for the uncertainty of sector c, we characterize the strong anisotropic electrons as beam-like when they are detected streaming mainly along the opposite direction of the IMF. Therefore, the in situ energetic electrons are probably accelerated in a confined site on the Sun, e.g., flaring AR, rather than a wide-spread CME-driven shock, and then traveling along open-field lines which connect the spacecraft to the acceleration region in a negative polarity. 

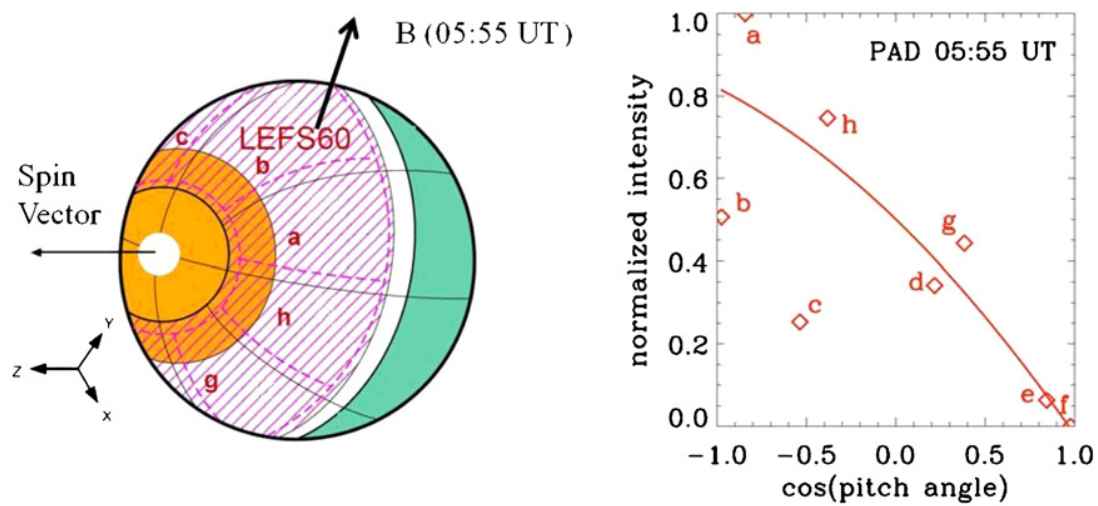

Figure 2. Left: schematic view of the $A C E$ /EPAM with the LEFS60 projected onto a unit sphere (adapted from Gold et al. 1998), and the magnetic field vector derived from the $A C E / M A G$ in GSE coordinates. Right: the normalized PAD at the peak time of the electron intensities in energy channel of $42-65 \mathrm{keV}$.

(A color version of this figure is available in the online journal.)

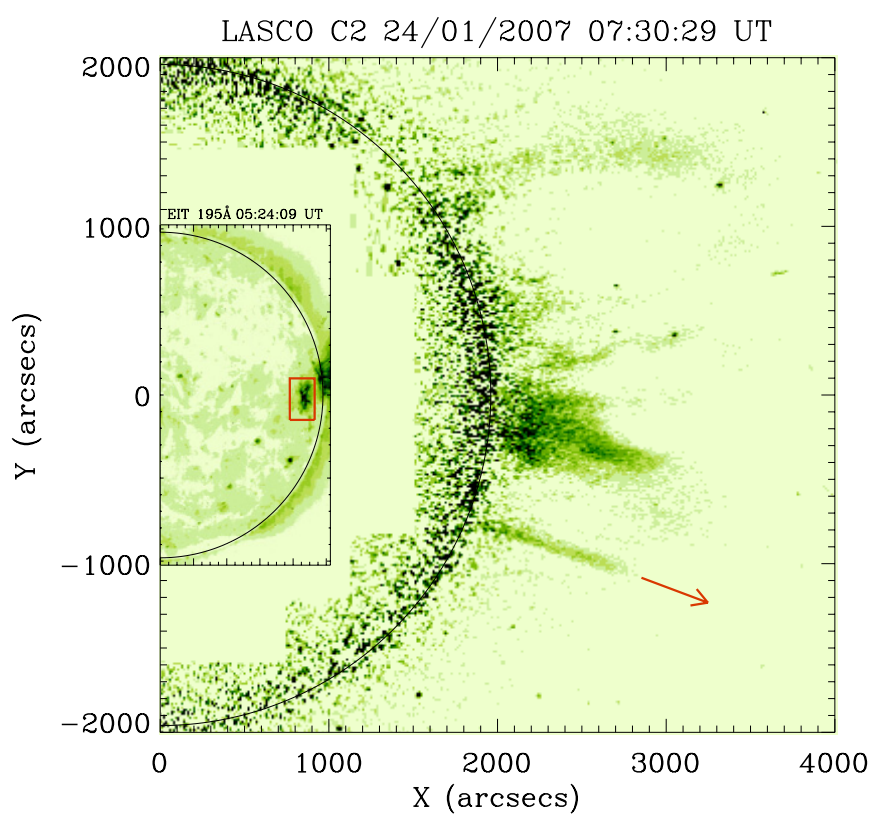

Figure 3. Composite data in WL and EUV images obtained from the $\mathrm{SOHO} /$ LASCO C2 and the $\mathrm{SOHO} / \mathrm{EIT}$, respectively. AR 10939 is marked by the red box, and a WL jet signature by the red arrow. A faint CME is observed to be lifting off from the nearby AR 10938, which is just behind the limb.

(A color version of this figure is available in the online journal.)

\section{REMOTE-SENSING OBSERVATIONS}

To identify the solar source of the beam-like energetic electrons, we first study the full-disk $195 \AA$ images from the Extreme-Ultraviolet Imaging Telescope (EIT; Delaboudinière et al. 1995), as well as the WL observations from the Large Angle and Spectrometric Coronagraph (LASCO; Brueckner et al. 1995) on board the Solar and Heliospheric Observatory $(\mathrm{SOHO})$. As shown in Figure 3, two western ARs were responsible for the noticeable coronal activities in the time window of the electron event: AR 10938, located at the far west limb, produced a faint CME with an angular width of $67^{\circ}$ and a projected speed of $295 \mathrm{~km} \mathrm{~s}^{-1}$. AR 10939, located at S06W63 and marked by the red box, produced the impulsive B7.3 flare and a jet which probably extended to a few solar radii observed by the LASCO C2 coronagraph and marked by the red arrow.
By applying running difference to the $\mathrm{C} 2$ images, the $\mathrm{WL}$ jet signature propagates to the southwest at an evaluated speed of $\sim 190 \mathrm{~km} \mathrm{~s}^{-1}$.

To further confirm the origin of the WL jet signature, EUV observations with high temporal cadence and spatial resolution are introduced. Figure 4 shows the development of a jet eruption from the AR 10939. In panel (a), the $171 \AA$ image at the peak time of the B7.3 flare is obtained from the Transition Region and Coronal Explorer (TRACE; Handy et al. 1999). To enhance the spatial structure, we apply a high-pass filter by subtracting a smoothed image from the original one. The hard X-ray (HXR) source is reconstructed by the Reuven Ramaty High Energy Solar Spectroscopic Imager (RHESSI; Lin et al. 2002) and co-aligned with the TRACE data. Red contour lines indicate the $25-50 \mathrm{keV}$ nonthermal bremsstrahlung source integrated from 05:16 to 05:17 UT. It is found the HXR source is located in a compact flaring region where the jet erupted nearby to the eastern large-scale loop structure. Panels (b)-(d) consist of running-difference images illustrating the jet evolution. The EUV jet propagates to southwest at an evaluated speed of $\sim 205 \mathrm{~km} \mathrm{~s}^{-1}$ during 05:16-05:25 UT. A simple estimation of the spatial and temporal correlation suggests that the WL jet signature seen with LASCO is the counterpart of the EUV jet, which erupted from AR 10939. The velocity of the jet slightly decreased at $\sim 3 R_{s}$.

The jet was also observed by the EUV Imaging Spectrometer (EIS; Culhane et al. 2007) on board the Hinode satellite. A raster scan using a $1^{\prime \prime}$ slit was performed with the EIS from 05:22 to $05: 44$ UT. The jet signature is seen in the $255.95-256.64 \AA$ intensity map in the left panel of Figure 5. The wave band consists of the main emission line He II peaked at $256.32 \AA$ and a blended one $\mathrm{Si}$ x at $258.37 \AA$ A. We do a two-component Gaussian fit and decompose it into two intensity maps, respectively, shown in the right panel of Figure 5. The compact flaring region where the jet erupted is shown in both of the two intensity maps. However, the jet itself is only detected in the hot coronal line Si $\mathrm{x}$ rather than the cool line He II, suggesting the jet is actually formed in a coronal site.

The compact flaring region where the jet erupted is further analyzed using the X-Ray Telescope (XRT; Golub et al. 2007) on board the Hinode satellite. Figure 6 shows the XRT image in Al_poly filter whose temperature response peaks at $7 \mathrm{MK}$. It is, after co-alignment, overlaid with the magnetic field polarities obtained from the Michelson Doppler Imager (MDI; Scherrer et al. 1995) on board $\mathrm{SOHO}$. The compact flaring region then 


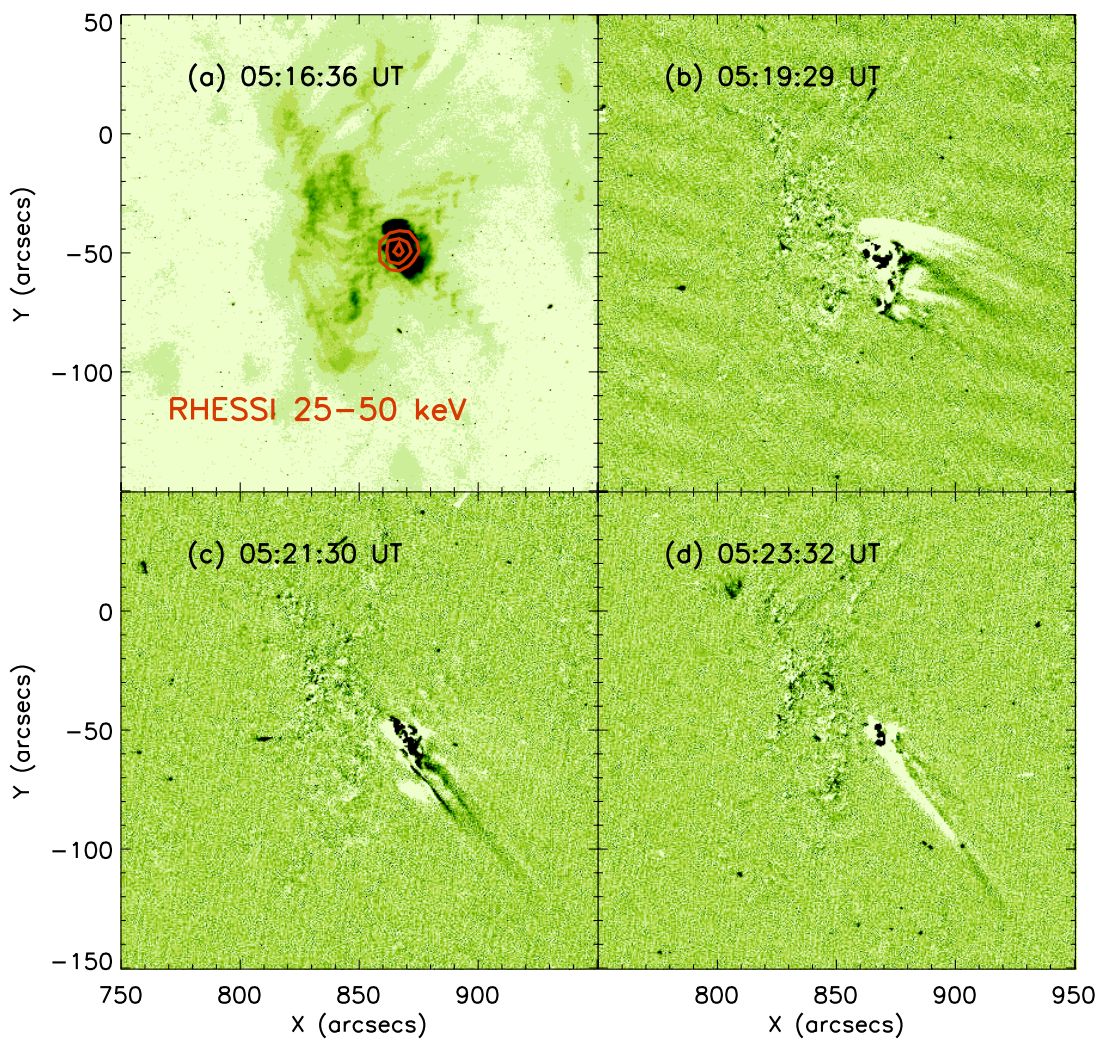

Figure 4. Jet eruption observed in TRACE $171 \AA$ Amages. (a) High-pass filtered image at the peak time of the B7.3 flare overlaid with the RHESSI HXR source in the energy channel of 25-50 keV. Panels (b)-(d) consist of running-difference images illustrating the evolution of the jet.

(A color version of this figure is available in the online journal.)
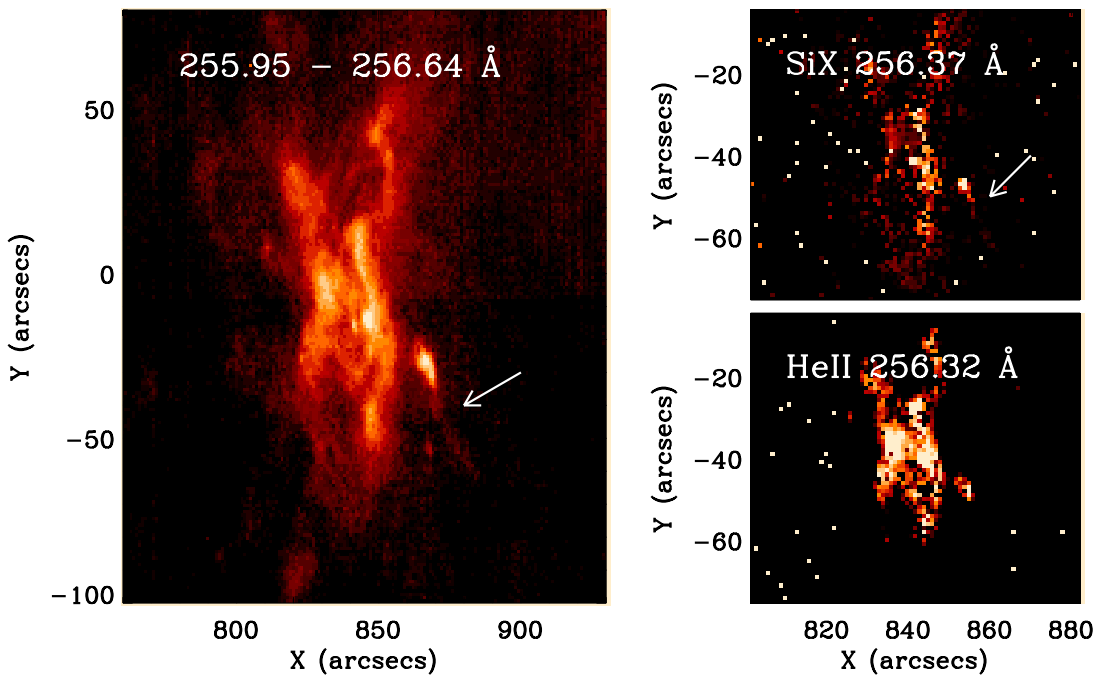

Figure 5. Jet signature observed in Hinode/EIS 255.95-256.64 Å intensity map. After a two-component Gaussian fit, the jet, marked by the white arrow, is only detectable by a blended emission line Si X.

(A color version of this figure is available in the online journal.)

is identified as a loop-like and cusp structure straddled on the right inversion line. We also mark the footpoints of open-field lines calculated using the potential-field source-surface model (PFSS; Schrijver \& DeRosa 2003). It is derived at 00:04 UT and rotated to $05: 22 \mathrm{UT}$. The open-field lines are rooted in an area of negative polarity nearby to the jet site. Therefore, the jet is probably produced by the reconnection between closed loops straddled on the middle inversion line and the western open-field lines.

\section{RELATING TO THE SOLAR SOURCE}

Based on the above analysis, the coronal jet is expected to be closely related to the in situ energetic electrons. The magnetic configuration, on the other hand, plays a crucial role in triggering jet eruption and guiding energetic particles from the acceleration region to interplanetary space. As shown in Figure 6, the magnetogram in the vicinity of AR 10939 is quadrupolar and potentially favorable for jet formation. It is, 


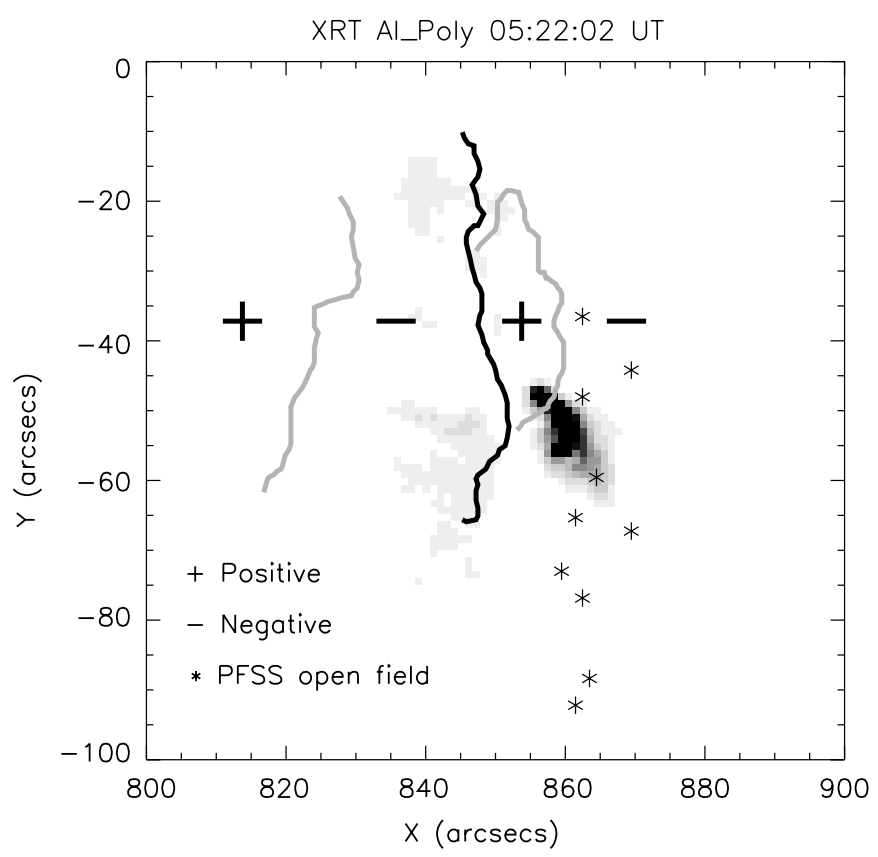

Figure 6. Hinode /XRT image by the Al_poly filter overlaid with the magnetic field polarities obtained from the $S O H O / \mathrm{MDI}$, and the footpoints of the PFSS modeled open-field lines. Inversion lines are marked by the black and gray lines. "+" indicates positive longitudinal magnetic field and "-" negative. "*” indicates footpoints of the open fields.

however, not possible to simulate a precise coronal magnetic configuration at the solar limb due to the projection. The EIS velocity map, from another perspective, gives us a clue to understand the magnetic topology. It is widely accepted that the redshifted downflows in ARs are observed in closed loops. The blueshifted outflows, usually persist at the edges of ARs, are variously interpreted as being due to magnetic funnels playing a role in coronal plasma circulation (Marsch et al. 2008), expansion of large-scale loops that lie over ARs (Harra et al. 2008), and reconnection along the quasi-separatrix layers where strong gradients of magnetic connectivity are present (Baker et al. 2009).

Figure 7 shows the EIS Fe XII emission line velocity map with magnetic contours from MDI and schema of a proposed coronal magnetic topology. Doppler velocities are determined by fitting a Gaussian function to the calibrated spectral profile. Blueshifts (redshifts) correspond to negative (positive) Doppler velocities in a range of $\pm 25 \mathrm{~km} \mathrm{~s}^{-1}$. Black contour lines indicate positive longitudinal magnetic field, and green negative. The strong blueshifted outflows might be explained by either of the mechanisms aforementioned. The redshifted area corresponds to closed loops of the AR 10939 marked by the red arcs. The jet is likely to be produced by reconnection between closed loops and negative open-field lines predicted by the PFSS model and marked by the black solid lines, along which accelerated particles escape into interplanetary space to be detected by the near-Earth spacecraft. Note that the in situ electron PAD (shown in Figure 2) is consistent with the open-field lines being of negative polarity. A CME lifted off from the AR 10938 is marked by the black dashed lines, which might compress the open-field lines to facilitate reconnection with the closed loops and the jet eruption (see the discussion in Section 5).

Timing information gives us another clue to relate to the solar source. Figure 8 shows the comparison of electron injection, jet duration, and the multi-wavelength flare emission. The electrons are released from the Sun at 05:19 UT \pm 5 minutes (see Section 2) and marked by the orange bar. The EUV jet duration lasted from 05:16 UT to 05:25 UT (see Section 3) and marked by the red bar. The temporal profiles are shown by GOES 1-8 ^ SXR, RHESSI 12-50 keV HXR, and the RSTN/ Learmonth $245 \mathrm{MHz}$ radio emission, respectively. The WIND/ WAVES radio dynamic spectra in the frequency range of 20 $\mathrm{kHz}$ to $14 \mathrm{MHz}$ show clear type-III bursts, which are generated by electron beams as they propagate along magnetic field lines from the solar corona to interplanetary medium. It is evident that the electron solar release has a good temporal correlation with the jet eruption, flare emission, and the consequent type-III radio bursts, suggesting a physical link with magnetic reconnection, jet production, high-energy emission, and the particle acceleration.

\section{DISCUSSION AND SUMMARY}

Table 1 summarizes the impulsive electron events and west hemisphere solar activities observed on 2007 January 24. The well-connected AR 10939 produced three jets identified in EUV images from the TRACE and STEREO/EUVI, respectively. The first jet erupted at $\sim 00: 35$ UT was associated with an HXR flare detectable from the RHESSI and type-III radio bursts from the WIND/WAVES. It is related to the electron event 1 whose solar release time is deduced at $\sim 00: 37 \mathrm{UT}$, consistent with the jet eruption and flare emission. The second jet erupted at

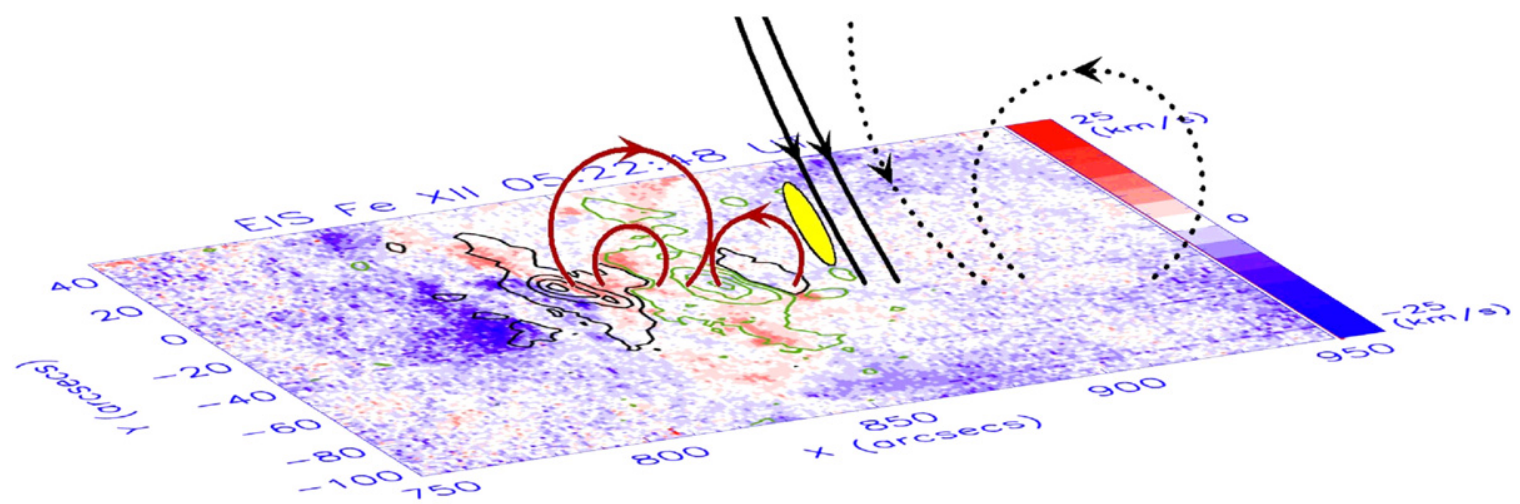

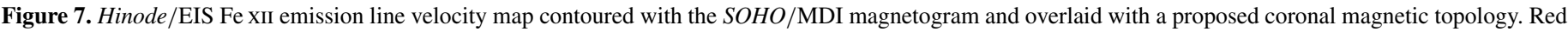

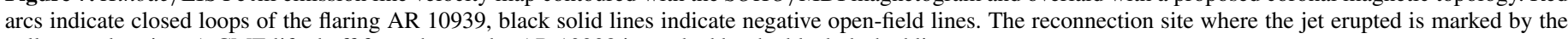
yellow oval region. A CME lifted off from the nearby AR 10938 is marked by the black dashed lines.

(A color version of this figure is available in the online journal.) 
Table 1

Electron Events and West Hemisphere Solar Activities on 2007 January 24

\begin{tabular}{|c|c|c|c|c|c|c|}
\hline \multirow{3}{*}{$\begin{array}{l}\text { SEP } \\
\text { (UT) }\end{array}$} & \multicolumn{3}{|c|}{ AR 10939} & \multicolumn{3}{|c|}{ AR 10938} \\
\hline & \multirow{2}{*}{$\begin{array}{c}\text { Jet } \\
(\mathrm{UT})\end{array}$} & \multirow[t]{2}{*}{ HXR Flare } & \multirow[t]{2}{*}{ Type-III Burst } & \multicolumn{3}{|c|}{$\mathrm{CME}$} \\
\hline & & & & $\begin{array}{c}\text { Liftoff } \\
\text { (UT) }\end{array}$ & $\begin{array}{l}\text { Width } \\
\text { (deg) }\end{array}$ & $\begin{array}{c}\text { Speed } \\
\left(\mathrm{km} \mathrm{s}^{-1}\right)\end{array}$ \\
\hline \multirow[t]{3}{*}{$00: 37$} & $00: 35^{\mathrm{a}}$ & Y (B5.5) & Y & $\ldots$ & $\ldots$ & $\ldots$ \\
\hline & $01: 15^{\mathrm{a}}$ & $\mathrm{N}$ & $\mathrm{N}$ & $\ldots$ & $\ldots$ & $\ldots$ \\
\hline & - & - & - & $05: 13$ & 67 & 295 \\
\hline \multirow[t]{2}{*}{ 05:19 } & $05: 16^{\mathrm{b}}$ & Y (B7.3) & Y & $\ldots$ & $\ldots$ & $\ldots$ \\
\hline & - & - & - & $15: 47$ & 72 & 381 \\
\hline
\end{tabular}

Notes.

-: No jet, HXR flare, or type-III burst observed. ...: No CME observed.

a Jets were identified by STEREO/EUVI in 10 minute temporal cadence.

${ }^{\mathrm{b}}$ Jet was identified by TRACE in $\sim 1$ minute cadence.
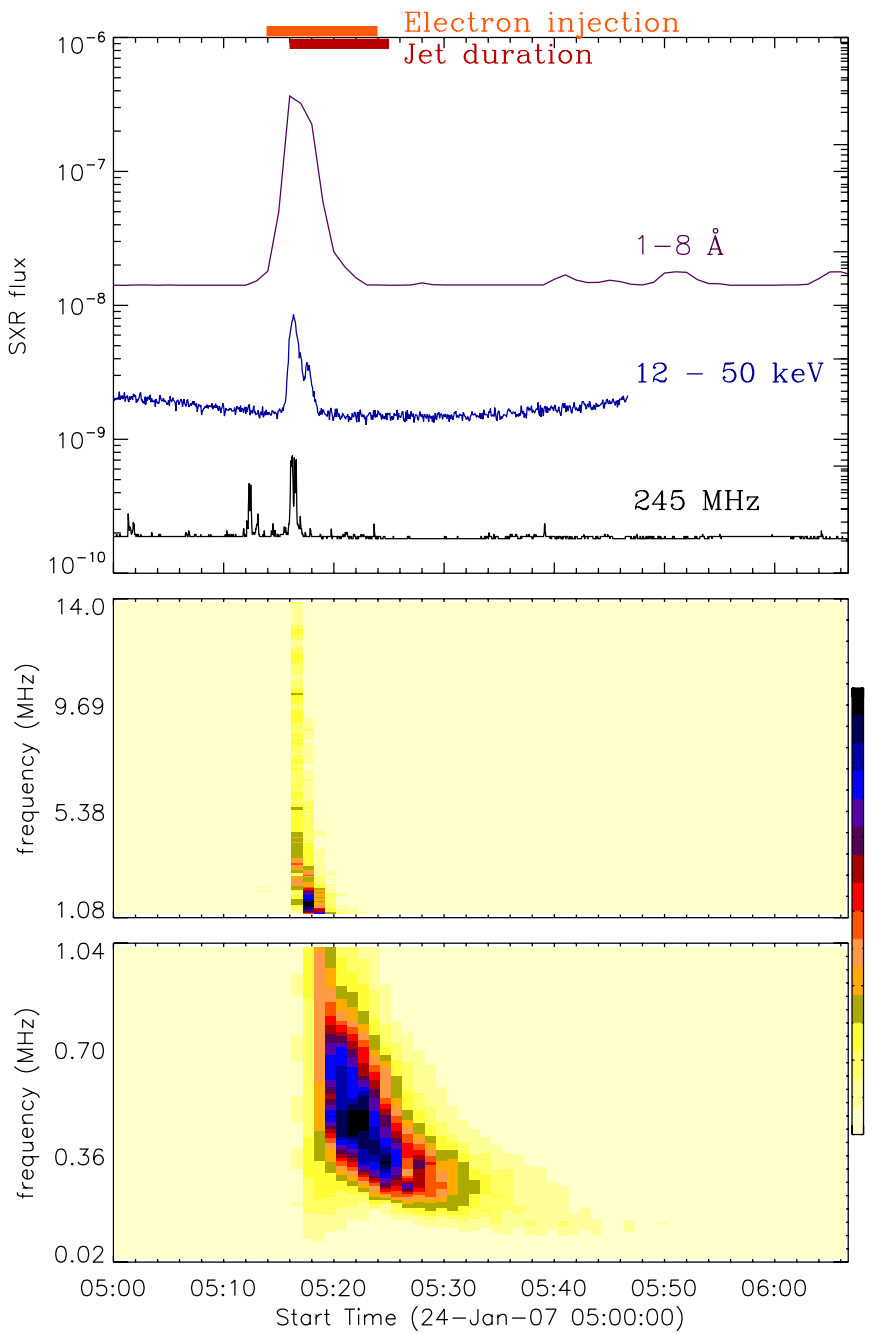

Figure 8. Electron injection compared with the jet eruption and multiwavelength flare emission. From top to bottom: light curves of GOES SXR 1-8 A, RHESSI HXR 12-50 keV, RSTN/Learmonth $245 \mathrm{MHz}$ radio emission, and the WIND/WAVES radio spectrograms at frequency range of $20 \mathrm{kHz}$ to $14 \mathrm{MHz}$.

(A color version of this figure is available in the online journal.)

$\sim 01: 15$ UT without HXR or radio signature. No corresponding electron flux enhancement was recorded by the in situ spacecraft. The third jet and the closely related electron event 2 were comprehensively analyzed above.
The far-side AR 10938 produced two CMEs (obtained from the LASCO CME catalog: http://cdaw.gsfc.nasa.gov/CME_list/ index.html). The first CME with an angular width of $67^{\circ}$ and a projected speed of $295 \mathrm{~km} \mathrm{~s}^{-1}$ lifted off at $\sim 05: 13 \mathrm{UT}$, close in time to the jet eruption at $\sim 05: 16 \mathrm{UT}$ and the electron solar release at $\sim 05: 19$ UT. We interpret that this CME might either facilitate the reconnection occurrence and jet eruption, or further accelerate particles in a higher coronal site via three-dimensional reconnection with the open fields. The second $\mathrm{CME}$, with an even larger angular width of $72^{\circ}$ and a higher plane-of-sky speed of $381 \mathrm{~km} \mathrm{~s}^{-1}$ lifted off at $\sim 15: 47 \mathrm{UT}$, however, was not temporally related to an SEP event. Furthermore, no type-II radio bursts were recorded in the time window of the impulsive electron events. Therefore, the jets in association with HXR flares and type-III radio bursts, rather than the slow and partial CMEs, are the crucial contributors to the interplanetary electron streams.

We combine a wide range of data sets, specifically in situ electron measurements and remote-sensing solar observations, to investigate the acceleration source of the impulsive electron events. Data analysis shows that (1) the electron solar release has a good temporal correlation with the jet eruption and the multiwavelength flare emission, namely SXR, HXR, and type-III radio burst. (2) The jet originated in a well-connected AR whose magnetic polarity structure favors the eruption, then formed in a coronal site, and extended to a few solar radii. (3) The PFSS modeled open fields are rooted in a negative polarity, along which energetic particles escape from the flaring AR to the near-Earth space, consistent with the in situ electron PAD. The results above confirm the viewpoint that the coronal jet is probably a key to understanding the production of interplanetary electron streams. However, many impulsive SEP events are not associated with jets or even lacking X-ray signatures (Nitta et al. 2008; Mason et al. 2009). The acceleration possibly takes place in the high corona via three-dimensional reconnection or other energizing processes.

We would like to emphasize that the magnetic topology plays a crucial role in triggering jet eruption and guiding particle transport. Based on the EIS velocity map, MDI magnetogram and the potential-field extrapolation, we propose a coronal field configuration relating the impulsive SEP event to the solar source. Detailed reconstruction of magnetic fields, unavailable to the present study, should be further introduced to explore this topic.

The authors thank the WIND/3DP/WAVES, ACE/EPAM/ MAG,Hinode/EIS/XRT,SOHO/EIT/MDI/LASCO,STEREO/ 
EUVI, RSTN/Learmonth, RHESSI, and TRACE teams for providing the data used in this study. The authors also thank the anonymous referee for valuable comments. This work was supported by the rolling grant from Science \& Technology Facilities Council (STFC) of the UK. L.vD.G. acknowledges funding through the Hungarian Science Foundation grant OTKA K81421, and the European Community's FP7/2007-2013 program through the SOTERIA Network (EU FP7 Space Science Project No. 218816).

\section{REFERENCES}

Baker, D., van Driel-Gesztelyi, L., Mandrini, C. H., Démoulin, P., \& Murray, M. J. 2009, ApJ, 705, 926

Brueckner, G. E., et al. 1995, Sol. Phys., 162, 357

Culhane, J. L., et al. 2007, Sol. Phys., 243, 19

Delaboudinière, J.-P., et al. 1995, Sol. Phys., 162, 291

Gold, R. E., et al. 1998, Space Sci. Rev., 86, 541

Golub, L., et al. 2007, Sol. Phys., 243, 63

Haggerty, D. K., \& Roelof, E. 2003, Adv. Space Res., 32, 423

Handy, B. N., et al. 1999, Sol. Phys., 187, 229

Harra, L. K., Sakao, T., Mandrini, C. H., Hara, H., Imada, S., Young, P. R., van Driel-Gesztelyi, L., \& Baker, D. 2008, ApJ, 676, L147
Kahler, S. W., Reames, D. V., \& Sheeley, N. R., Jr. 2001, ApJ, 562, 558

Kallenrode, M.-B. 2003, J. Phys. G: Nucl. Part. Phys., 29, 965

Krucker, S., Larson, D. E., Lin, R. P., \& Thompson, B. J. 1999, ApJ, 519, 864

Li, C., Owen, C. J., Matthews, S. A., Dai, Y., \& Tang, Y. H. 2010, ApJ, 720, L36

Lin, R. P. 1995, Space Sci. Rev., 71, 125

Lin, R. P., et al. 2002, Sol. Phys., 210, 3

Marsch, E., Tian, H., Sun, J., Curdt, W., \& Wiegelmann, T. 2008, ApJ, 685, 1262

Mason, G. M. 2007, Space Sci. Rev., 130, 231

Mason, G. M., Nitta, N. V., Cohen, C. M. S., \& Wiedenbeck, M. E. 2009, ApJ, 700, L56

Nitta, N. V., Mason, G. M., Wiedenbeck, M. E., Cohen, C. M. S., Krucker, S., Hannah, I. G., Shimojo, M., \& Shibata, K. 2008, ApJ, 675, L125

Parker, E. N. 1958, ApJ, 128, 664

Pick, M., Mason, G. M., Wang, Y.-M., Tan, C., \& Wang, L. 2006, ApJ, 648, 1247

Reames, D. V. 1999, Space Sci. Rev., 90, 413

Scherrer, P. H., et al. 1995, Sol. Phys., 162, 129

Schrijver, C. J., \& DeRosa, M. L. 2003, Sol. Phys., 212, 165

Shibata, K., Nitta, N., Strong, K. T., Matsumoto, R., Yokoyama, T., Hirayama, T., Hudson, H., \& Ogawara, Y. 1994, ApJ, 431, L51

Wang, Y.-M., Pick, M., \& Mason, G. M. 2006, ApJ, 639, 495 